\title{
BBN as Probe of Fundamental Physics
}

\author{
L. A. Popa and A. Caramete \\ ISS/Institute for Space Sciences Bucharest-Magurele, \\ Romania
}

\section{Introduction}

The primordial Universe provides a laboratory to probe fundamental physics at high energies. Relics from those early epochs, such as the light elements synthesized during primordial Big Bang Nucleosynthesis (BBN) (Burles et al., 2001; Eidelman et al., 2004; Olive et al., 2000; Wagoner et al., 1967) when the Universe was only a few minutes old, and the Cosmic Microwave Background ( $\mathrm{CMB}$ ) photons, last scattered when the protons and electrons recombined some 400 thousand years later, represent powerful probes of the high energy phenomena pointing beyond the standard models of cosmology, particle physics and general relativity (Boesgaard \& Steigman, 1985; Steigman et al., 1977; Steigman, 2007).

During its earlier evolution the Universe was hot and dense. The combination of high temperature and density ensures that collision rates are very high during early epochs, guaranteeing that all particles were in equilibrium at sufficiently early times. As the Universe expands and cools, interaction rates decline and, depending on the strength of their interactions, different particles depart from equilibrium at different epochs.

At a temperature of few $\mathrm{MeV}$, the neutrino interaction rates become lower than the Hubble expansion rate, decoupling from the $\mathrm{CMB}$ photons and $e^{ \pm}$pairs present at that time. However, electron neutrinos (and antineutrinos) continue to interact with the baryons through the weak interactions until the Universe where few seconds old and the temperature has dropped below $\mathrm{MeV}$. The interactions among neutrons, protons and $e^{ \pm}$continue to influence the ratio of neutron to proton number densities, tracking its equilibrium value. After electrons, neutrons and protons combine to form neutral atoms at recombination, the CMB photons propagate freely. This occurs when the Universe is some 400 thousand years old. The relic photons from this epoch redshifted to the currently observed CMB black body temperature $T_{c m b}=2.725 \mathrm{~K}$.

Predictions of the abundances of the light elements as $\mathrm{D},{ }^{3} \mathrm{He},{ }^{4} \mathrm{He}$ and ${ }^{7} \mathrm{Li}$ synthesized at the BBN epoch are in good agreement with the primordial abundances inferred from observational data, which validates the Standard Big Bang Nucleosynthesis (SBBN).

The primordial abundances of the relic nuclei produced during SBBN depend on the baryon number density and on the Hubble expansion rate of the Universe. At the same time, the shape and amplitude of the $\mathrm{CMB}$ anisotropy angular power spectra at the time of recombination depend on the same parameters. One of the key cosmological tests in understanding the cosmological dynamics between BBN and recombination is the determination of baryon energy density mass fraction, $\Omega_{b} h^{2}$, at these two different epochs.

The abundance of baryons, $\eta_{10}$, is related to the number density of baryons, $n_{B}$, and the number density of CMB photons, $n_{\gamma}$, through: $\eta_{10}=10^{10}\left(n_{B} / n_{\gamma}\right)$. The baryon energy density 
mass fraction influences the growth rate of the density perturbation through its impact on the Hubble expansion rate. It is convenient to express $\eta_{10}$ in terms of this two parameters as: $\eta_{10}=274 \Omega_{b} h^{2}$, where $h$ is the reduced Hubble constant at the present time $\left(h=H_{0} / 100 \mathrm{~km}\right.$ $\left.\mathrm{s}^{-1} \mathrm{Mpc}^{-1}\right)$. During expansion of the Universe, $n_{B}$ and $n_{\gamma}$ decrease but remain unchanged in a given comoving volume. Therefore, the value of $\eta_{10}$ measured at BBN epoch and at recombination epoch should be the same.

Among the nuclides synthesized at the BBN epoch, deuterium is the better indicator of the primordial baryon abundance since no significant amounts of deuterium are synthesized after BBN (Epstein et al., 1976). The measurement of the QSO Absorption Lines Systems (QSOALS) leads to the determination of the primordial deuterium abundance (Pettini et al., 2008) from which the infered baryon abundance is $\eta_{10}(B B N)=5.80_{-0.28}^{+0.27}\left(\Omega_{b} h^{2}=0.0212 \pm 0.0010\right)$.

The analysis of WMAP 7-year data (Komatsu et al., 2009) provides an independent determination of the baryon abundance $\eta_{10}(C M B)=6.190 \pm 0.145\left(\Omega_{b} h^{2} 0.02260 \pm 0.00053\right)$.

The two independent determinations are in good agreement, differing by only $1.3 \sigma$.

BBN also provides powerful constraints on possible deviations from the standard cosmology and on new physics beyond the Standard Model (SM) particle physics (Sarkar, 1996). Many non-SBBN models introduce new free parameters in addition to the baryon energy density parameter. Most of these models assume either non-standard contribution to the total energy density, or a lepton asymmetry. This paper aims to place constraints on parameters of two types of non-SBBN models by using most of the present cosmological data complemented with the BBN predictions on the ${ }^{4} \mathrm{He}$ abundance.

These models include: i) the leptonic asymmetric cosmological models, challenging the standard neutrino sector and ii) the Higgs inflation models, challenging the electroweak sector of the SM of particle physics.

\section{Challenging the neutrino physics}

The radiation budget of the Universe relies on a strong theoretical prejudice: apart from the CMB photons, the relativistic background would consist of neutrinos and of possible contributions from other relativistic relicts. The main constraints on the radiation energy density come either from the very early Universe, where the radiation was the dominant source of energy, or from the observation of cosmological perturbations which carry the information about the time equality between matter and radiation.

The primordial abundance of the light elements depends also on the radiation energy density at the BBN epoch (energy density of order $\mathrm{MeV}^{4}$ ), usually parametrized by the effective number of relativistic neutrino species, $N_{e f f}$. Meanwhile, the number of active neutrino flavors have been fixed by $Z^{0}$ boson decay width to $N_{v}=2.944 \pm 0.012$ (Eidelman et al., 2004), while the combined study of the incomplete neutrino decoupling and the QED corrections indicate that the number of relativistic neutrino species is $N_{e f f}=3.046$ (Mangano et al., 2002). Any departure of $N_{e f f}$ from this last value would be due to non-standard neutrino features or to the contribution of other relativistic relics, having as main effect the modification of the competition between the nuclear reaction rates and the Hubble expansion rate. Since the primordial ${ }^{4} \mathrm{He}$ mass fraction, $Y_{P}$ is largely determined by the neutron to proton ratio, $Y_{P}$ is quite sensitive to the competition between the weak interaction rates and the expansion rate. 
The most natural phenomenological extension of the standard neutrino sector is the consideration of the leptonic asymmetry (Freese et al., 1983; Ruffini et al., 1983; 1988), parametrized by the neutrino degeneracy parameter $\xi_{v}=\mu_{v} / T_{v_{0}}\left[\mu_{v}\right.$ is the neutrino chemical potential and $T_{v_{0}}$ is the present temperature of the neutrino background, $T_{v_{0}} / T_{\mathrm{cmb}}=$ $(4 / 11)^{1 / 3}$ ]. Although the standard model of particle physics predicts the value of leptonic asymmetry of the same order as the value of the baryonic asymmetry, $B \sim 10^{-10}$, there are many particle physics scenarios in which a leptonic asymmetry much larger can be generated (Chu \& Cirelli, 2006; Smith et al., 2006).

One of the cosmological implications of a larger leptonic asymmetry is the possibility to generate baryonic asymmetry of the Universe through the sphaleron processes (Buchmuller et al., 2004; Falcone \& Tramontano, 2001; Kuzmin et al., 1985). Therefore, distinguishing between a vanishing and non-vanishing $\xi_{v}$ at the BBN epoch is a crucial test of the standard assumption that sphaleron effects equilibrating the cosmic lepton and baryon asymmetries.

The measured neutrino mixing parameters implies that neutrinos reach the chemical equilibrium before BBN (Abazajian et al., 2002; Dolgov et al., 2002; Wong, 2002), so that all neutrino flavors are characterized by the same degeneracy parameter, $\xi_{v}$, at this epoch. The most important impact of the leptonic asymmetry on BBN is the shift of the beta equilibrium between protons and neutrons and the increase of the radiation energy density parametrized by:

$$
\Delta N_{e f f}\left(\xi_{v}\right)=3\left[\frac{30}{7}\left(\frac{\xi_{v}}{\pi}\right)^{2}+\frac{15}{7}\left(\frac{\xi_{v}}{\pi}\right)^{4}\right] .
$$

The total extra energy density can be splitted in two distinct uncorrelated contributions, first due to net lepton asymmetry of the neutrino background, $\Delta N_{e f f}\left(\xi_{v}\right)$, and second due to the extra contributions from other unknown processes, $\Delta N_{e f f}^{o t h}$ :

$$
\Delta N_{e f f}=\Delta N_{e f f}(\xi)+\Delta N_{e f f}^{o t h} .
$$

\subsection{Present bounds on lepton asymmetry and radiation energy density}

The BBN constraints on $\mathrm{N}_{\text {eff }}$ have been analyzed by comparing the theoretical predictions and experimental data on the primordial abundances of light elements, by using the baryon abundance derived from the WMAP 3-year CMB temperature and polarization measurements (Hinshaw et al., 2007; Page et al., 2007; Spergel et al., 2007): $\eta_{B}=6.14 \times 10^{-10}(1.00 \pm 0.04)$. In particular, the ${ }^{4} \mathrm{He}$ abundance, $Y_{p}$, is quite sensitive to the value of $N_{\text {eff }}$. The conservative error analysis of helium abundance, $Y_{P}=0.249 \pm 0.009$ (Olive \& Skillman, 2004), yielded to $N_{\text {eff }}=3.1_{-1.2}^{+1.4}$ (95\% CL) in good agreement with the standard value (Mangano et al., 2007), but still leaving some room for non-standard values.

More stringent error bars of helium abundance , $Y_{p}=0.2516 \pm 0.0011$ (Izotov et al., 2007), leaded to $N_{\text {eff }}=3.32_{-0.24}^{+0.23}(95 \% \mathrm{CL})$ and a degeneracy parameter $-0.04<\xi_{v}<0.07(68 \% \mathrm{CL})$ (Ichikawa et al., 2007; Serpico \& Raffelt, 2005).

The CMB anisotropies and LSS matter density fluctuations power spectra carry the signature of the energy density of the Universe at the time of matter-radiation equality (energy density of order $\mathrm{eV}^{4}$ ), making possible the measurement of $N_{\text {eff }}$ through its effects on the growth of cosmological perturbations. The number of relativistic neutrino species influences the 
$\mathrm{CMB}$ power spectrum by changing the time of matter-radiation equality that enhances the integrated Sachs-Wolfe effect, leading to a higher first acoustic Doppler peak amplitude. Also, the temperature anisotropy of the neutrino background (the anisotropic stress) acts as an additional source term for the gravitational potential (Hu et al., 1995; Trotta \& Melchiorri, 2005), changing the CMB anisotropy power spectrum at the level of $\sim 20 \%$.

The delay of the epoch of matter-radiation equality shifts the LSS matter power spectrum turnover position toward larger angular scales, suppressing the power at small scales.

In particular, the non-zero neutrino chemical potential leads to changes in neutrino free-streaming length and neutrino Jeans mass due to the increase of the neutrino velocity dispersion (Ichiki et al., 2007; Lattanzi et al., 2005).

A lower limit to $N_{\text {eff }}>2.3(95 \% \mathrm{CL})$ was recently obtained from the analysis of the WMAP 5-year (WMAP5) data alone (Dunkley et al., 2009), while the combination of the WMAP5 data with distance information from baryonic acoustic oscillations (BAO), supernovae (SN) and Hubble constant measured by Hubble Space Telescope (HST), leaded to $N_{\text {eff }}=4.4 \pm 1.5$ (68\% CL), fully consistent with the standard value (Komatsu et al., 2009).

The bounds on the radiation content of the Universe and neutrino properties obtained from the analysis of the WMAP-5 year CMB measurements complemented with most of the existing CMB and LSS measurements, with self-consistent constraints on the primordial helium abundance from BBN leaded to a mean value (at $68 \% \mathrm{CL}$ ) of the effective number of relativistic neutrino species of $N_{\text {eff }}=3.256_{-0.641}^{+0.607}$ and a neutrino degeneracy parameter of $-0.216 \leq \xi_{v} \leq 0.226$ (Popa \& Vasile, 2008).

\subsection{New bounds on neutrino properties: BBN and CMB constraints}

In this section we revisit the constraints on the lepton asymmetry and radiation energy density by using the latest cosmological and astrophysical measurements: the WMAP 7-year CMB measurements (Komatsu et al., 2011; Larson et al., 2011) complemented with geometric probes from the Type Ia supernovae (SN) distance-redshift relation, the baryon acoustic oscillations $(\mathrm{BAO})$ and the BBN predictions on $Y_{p}$.

The SN distance-redshift relation has been studied in detail in the recent unified analysis of the published heterogeneous SN data sets the Union Compilation08 (Kowalski et al., 2008; Riess et al., 2009).

The BAO in the distribution of galaxies are extracted from Two Degree Field Galaxy Redshift Survey (2DFGRS)the Sloan Digital Sky Surveys Data Release 7 (Percival et al., 2010).

The BBN predicted values of $Y_{P}$ are obtained by using the PArthENoPE code (Pisanti et al., 2008). Starting from nuclear statistical equilibrium conditions, the code determines $Y_{P}$ as function of $\Omega_{b} h^{2}, \Delta N_{e f f}^{\text {oth }}$ and $\xi_{v}$.

We use these measurements especially because we are testing models deviating from the standard Friedmann expansion. These datasets properly enables us to account for any shift of the $\mathrm{CMB}$ angular diameter distance and of the expansion rate of the universe. Hereafter, we will denote WMAP7+BAO+SNIa+BBN data set as WMAP7+BBN+All. We perform our analysis in the framework of the extended $\Lambda$ CDM cosmological model described by $6+3$ free parameters:

$$
\Theta=\{\underbrace{\Omega_{b} h^{2}, \Omega_{d m} h^{2}, H_{0}, z_{r e}, n_{s}, A_{s}}_{\text {standard }}, \Omega_{v} h^{2}, \xi_{v}, \Delta N_{e f f}^{o t h}\} .
$$



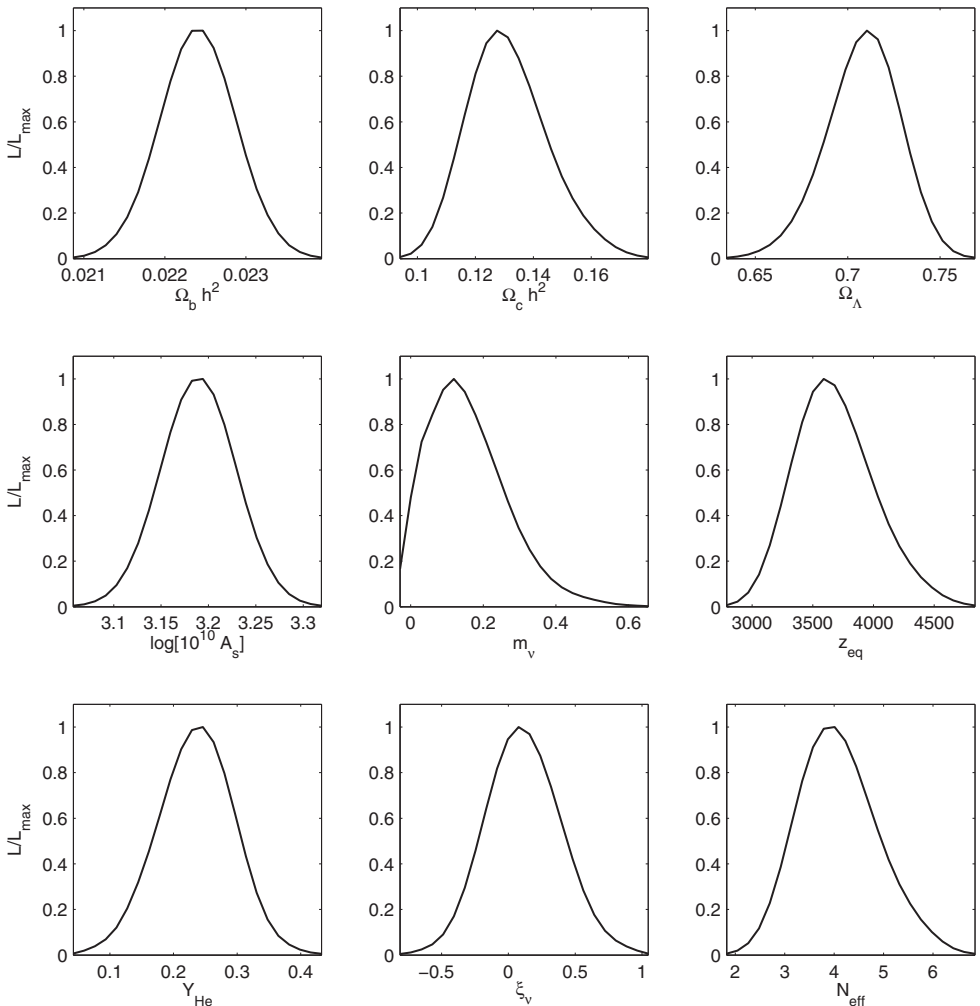

Fig. 1. The marginalized posterior likelihood probability distributions of the main cosmological parameters obtained from the fit of the leptonic asymmetric cosmological models to the WMAP7+BBN+All data set.

Here $\Omega_{b} h^{2}$ and $\Omega_{d m} h^{2}$ are the baryon and cold dark matter energy density parameters, $H_{0}$ is the Hubble expansion rate, $z_{r e}$ is the redshift of reionization, $n_{s}$ is the scalar spectral index of the primordial density perturbation power spectrum and $A_{S}$ is its amplitude at the pivot scale $k_{*}=0.002 \mathrm{hMpc}^{-1}$. The additional three parameters denote the neutrino energy density $\Omega_{v} h^{2}$, the neutrino degeneracy parameter $\xi_{v}$ and the contribution of extra relativistic degrees of freedom from other unknown processes $\Delta N_{o t h}^{e f f}$.

The likelihood probabilities are evaluated by using the public packages COSMOMC and CAMB (Lewis et al., 2000; Lewis \& S. Briddle, 2002) modified to include the formalism for the leptonic asymmetric cosmological models (Popa \& Vasile, 2008).

We assume uniform prior probability on parameters $\Theta$ and compute the cumulative distribution function $C(\theta)=\int_{\Theta_{\min }}^{\Theta} \mathcal{L}(\Theta) d \Theta / \int_{\Theta_{\min }}^{\Theta_{\max }} \mathcal{L} d \Theta$, quoting the upper and lower intervals at $68 \% \mathrm{CL}$.

Figure 1 presents the marginalized likelihood probabilities of the main cosmological parameters as obtained from the fit of the leptonic asymmetric cosmological models to the WMAP7+BBN+All data set and Figure 2 presents the degeneracies among them. 

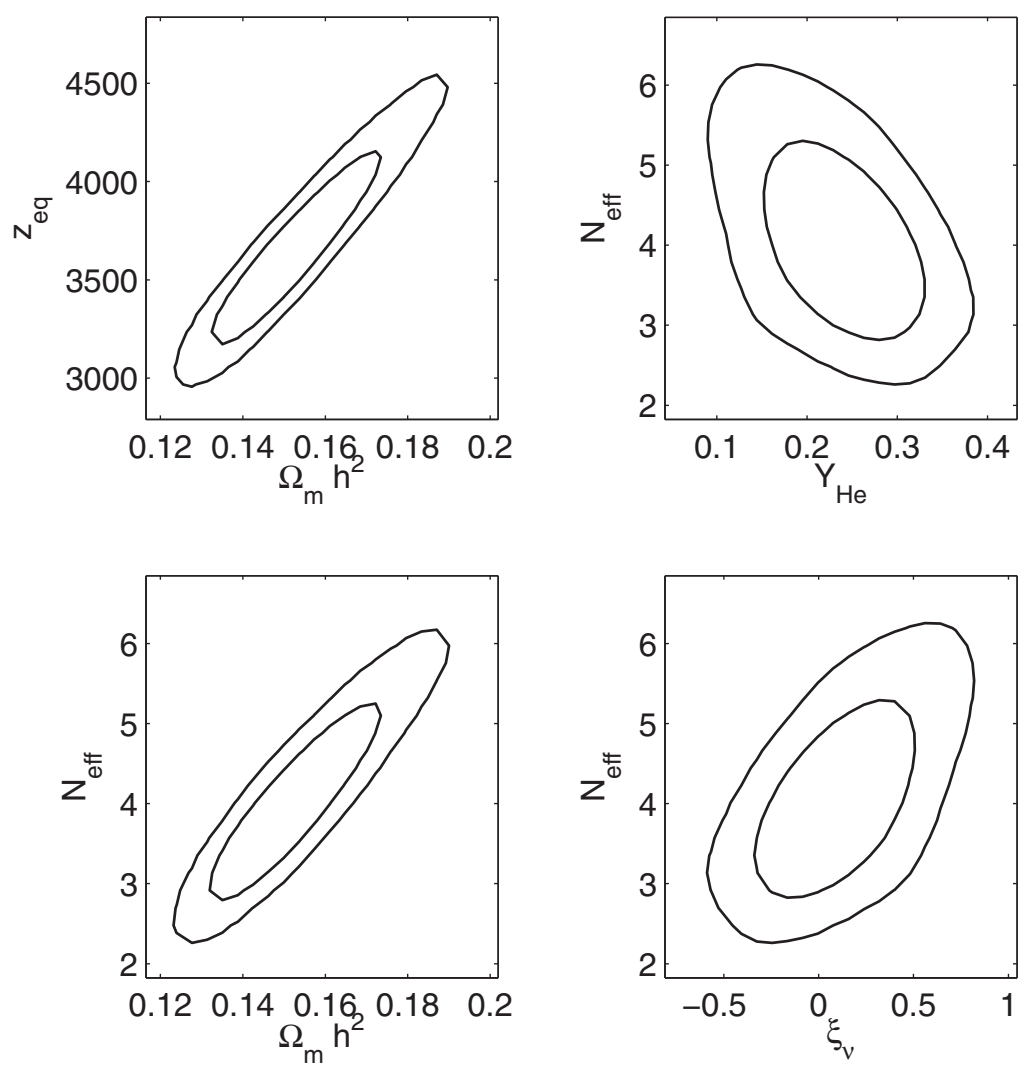

Fig. 2. Two dimensional joint probability distributions ( $68 \%$ and $95 \%$ CL) showing the degeneracy between different cosmological parameters as obtained from the fit of the leptonic asymmetric cosmological models to the WMAP7+BBN+All data set.

As neutrinos with $\mathrm{eV}$ mass decouple when they are still relativistic $\left(T_{d e c} \sim 2 \mathrm{MeV}\right)$, the main effect of including $\Delta N_{e f f}$ is the change of relativistic energy density. This changes the redshift of matter-radiation equality, $z_{e q}$, that affects the determination of $\Omega_{m} h^{2}$ from CMB measurements because of its linear dependence on $N_{\text {eff }}$ (Komatsu et al., 2009):

$$
1+z_{e q}=\frac{\Omega_{m} h^{2}}{\Omega_{\gamma} h^{2}} \frac{1}{1+0.2271 N_{e f f}} .
$$

Here $\Omega_{\gamma} h^{2}=2.469 \times 10^{-5}$ is the present photon energy density parameter for $T_{c m b}=2.725 \mathrm{~K}$. As a consequence, $N_{e f f}$ and $\Omega_{m} h^{2}$ are linearly correlated, with the width of degeneracy line given by the uncertainty in the determination of $z_{e q}$.

In Table 1 we compare the mean values and the absolute errors on the main cosmological parameters obtained from the analysis of WMAP5+BBN+All and WMAP7+BBN+All data sets. For all parameters, except $m_{v}$, we quote the errors at $68 \%$ CL. For $m_{v}$ we give the upper limits at 95\% CL. 
Parameter WMAP5+BBN+All WMAP7+BBN+All

\begin{tabular}{lll}
\hline & & \\
$\Omega_{b} h^{2}$ & $0.02246_{-0.00072}^{+0.00063}$ & $0.02240_{-0.00045}^{+0.00022}$ \\
$\Omega_{d m} h^{2}$ & $0.1115_{-0.0089}^{+0.0093}$ & $0.1135_{-0.0064}^{+0.0061}$ \\
$z_{r e}$ & $11.31_{-2.11}^{+1.92}$ & $10.96_{-0.83}^{+0.59}$ \\
$n_{s}$ & $0.965 \pm 0.018$ & $0.969 \pm 0.008$ \\
$\ln \left[10^{10} A_{s}\right]$ & $3.265 \pm 0.056$ & $3.189_{-0.019}^{+0.018}$ \\
$m_{v}(\mathrm{eV})$ & $\leq 0.535$ & $\leq 0.412$ \\
$\xi_{v}$ & $0.051 \pm 0.221$ & $0.037_{-0.143}^{+0.123}$ \\
$\Delta N_{\text {eff }}$ & $0.256_{-0.641}^{+0.607}$ & $0.209_{-0.401}^{+0.332}$ \\
$Y_{p}$ & $0.2487_{-0.0484}^{+0.0451}$ & $0.2356_{-0.0284}^{+0.0271}$ \\
$z_{\text {eq }}$ & $3124_{-128}^{+120}$ & $3132_{-87}^{+64}$ \\
& & \\
\hline \hline
\end{tabular}

Table 1. The table shows the mean values and the absolute errors on the main cosmological parameters obtained from the analysis of WMAP5+BBN+All and WMAP7+BBN+All data sets. For all parameters, except $m_{v}$, we quote the errors at $68 \%$ CL. For $m_{v}$ we give the upper limits at $95 \%$ CL.

From the analysis of WMAP7+BBN+All data we find a mean value of $N_{e f f}=3.21_{-0.401}^{+0.332}$, bringing an improvement over the similar result obtained from WMAP5+BBN+ALL data, $N_{\text {eff }}=3.26_{-0.690}^{+0.638}$. We also obtain improved values for ${ }^{4}$ He mass fraction, $Y_{p}=0.2356_{-0.0284}^{+0.0271}$, and neutrino degeneracy parameter, $-0.123 \leq \xi_{v} \leq 0.143$.

We find also a significantly reduced upper limit of the neutrino mass, $m_{v}<0.412 \mathrm{eV}$.

\section{Challenging the standard model of particle physics}

The primary goal of particle cosmology is to obtain a concordant description of the early evolution of the universe, establishing a testable link between cosmology and particle physics, consistent with both unified field theory and astrophysical and cosmological measurements.

Inflation is the most simple and robust theory able to explain the astrophysical and cosmological observations, providing at the same time self-consistent primordial initial condition mechanisms for the quantum generation of scalar (curvature) and tensor (gravitational waves) perturbations. In the simplest class of inflationary models, inflation is driven by a single scalar field $\phi$ (or inflaton) with some potential $V(\phi)$ minimally coupled to the Einstein gravity. The perturbations are predicted to be adiabatic, nearly scale-invariant and Gaussian distributed, resulting in an effectively flat universe.

The possibility that the Standard Model (SM) of particle physics with an additional non-minimally coupled term of the Higgs field to the gravitational Ricci scalar can give rise to inflation have been recently investigated by a number of authors (Barvinsky et al., 2008; Bezrukov \& Shaposhnikov, 2008; Bezrukov et al., 2009; De Simone et al., 2009). This scenario is based on the observation that the problem of the very small value of Higgs quadratic coupling required by the $\mathrm{CMB}$ anisotropy data can be solved if the Higgs inflaton has a large coupling to gravity. The resultant Higgs inflaton effective potential in the inflationary domain 

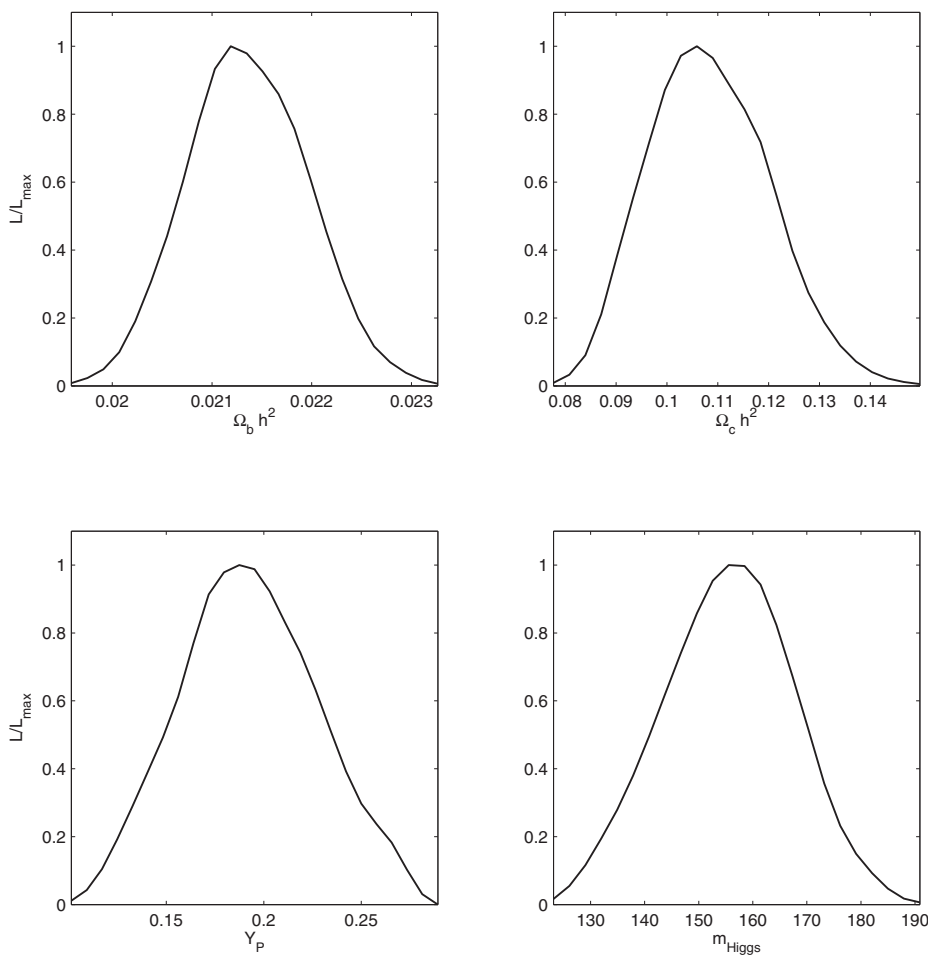

Fig. 3. The marginalized posterior likelihood probability distributions of the main cosmological parameters obtained from the fit of the Higgs inflation model to the WMAP7+BBN+All data set.

is effectively flat and can result in successful inflation for values of non-minimally coupling constant $\xi \sim 10^{3}-10^{4}$, allowing for cosmological values for Higgs boson mass in a window in which the Higgs vacuum expectation value $\langle v\rangle=246.22 \mathrm{GeV}$ is the minimum of the inflationary potential (Espinosa et al., 2008). The fluctuations of the electroweak vacuum expectation value might change the dynamics of the Higgs field during inflation, leading to modification of the main inflationary parameters and of the Higgs boson mass.

\subsection{BBN and the Higgs vacuum expectation value}

A modification of the Higgs vacuum expectation value during BBN leads to variations of the the neutron-proton mass difference, $\Delta m_{n p}$, the Fermi constant, $G_{F}$, the deuterium binding energy, $\epsilon_{D}$, and the electron mass, $m_{e}$.

The ${ }^{4} \mathrm{He}$ abundance is very sensitive to the parameters that fixed the neutron-to-proton ratio. In thermal equilibrium, this ratio is given by:

$$
\frac{Y_{n}}{Y_{p}}=e^{-\Delta m_{n p} / T}
$$


where $Y_{n}$ and $Y_{p}$ are the neutron and proton abundances and $T$ is the temperature in $\mathrm{MeV}$. The neutron-proton mass difference is affected by a change in the Higgs vacuum expectation value according to (Bergstrom et al., 1999):

$$
\frac{\delta \Delta m_{n p}}{\Delta m_{n p}}=1.587 \frac{\Delta<v>}{<v>}
$$

An increase in the Higgs vacuum expectation value $\langle v\rangle$ leads to an increase in $\Delta m_{n p}$. This produces a smaller neutron-to-proton equilibrium ratio and a smaller abundance of ${ }^{4} \mathrm{He}$ (Yoo \& Scherrer, 2003). On the other hand, a larger Higgs vacuum expectation value during BBN results in: i) a smaller value of the Fermi coupling constant, $G_{F}=1 / \sqrt{2}<v>^{2}$, leading to earlier freeze-out of the weak reactions and producing more ${ }^{4} \mathrm{He}$; ii) an increase in the electron mass, $m_{e}$, and a decrease of the weak reaction rates, producing also more ${ }^{4} \mathrm{He}$ (Landau et al., 2008).

The dependence of the deuterium binding energy, $\epsilon_{D}$, on the Higgs vacuum expectation value is extremely model dependent and can be approximated as (Yoo \& Scherrer, 2003):

$$
\frac{\Delta \epsilon_{D}}{\left(\epsilon_{D}\right)_{0}} \simeq \kappa \frac{\Delta<v>}{<v>_{0}}
$$

where $\kappa$ is a model dependent constant (e.g. $\kappa \simeq-6.230$ for a Reid potential). An increase in the Higgs vacuum expectation value results in a decrease in the deuterium binding energy, leading to a smaller initial deuterium abundance (Landau et al., 2008):

$$
Y_{d}=\frac{Y_{n} Y_{p} e^{11.605 \epsilon_{D} / T_{9}}}{0.471 T_{9}^{3 / 2}}
$$

where $T_{9}$ is the temperature in units of $10^{9} \mathrm{~K}$, and $\epsilon_{D}$ is in $\mathrm{MeV}$. The production of ${ }^{4} \mathrm{He}$ begins later, leading to a smaller helium abundance and an increase in the deuterium abundance (Yoo \& Scherrer, 2003).

The electron mass is proportional to the Higgs vacuum expectation value:

$$
\frac{\Delta m_{e}}{m_{e}}=\frac{\Delta<v>}{<v>} .
$$

A value of electron mass during BBN different from the present one translates into changes of electron and positron energy densities, leading to modifications of the Hubble expansion rate. Also, an increase of the electron mass during BBN slowers the neutron-proton intreaction rates, leading to a higher ${ }^{4} \mathrm{He}$ abundance. It is important to note that the modification of the primordial abundances due to the changes of the weak interaction rates are dominant over those due to the changes in the Hubble expansion rate (Yoo \& Scherrer, 2003).

\subsection{Bounds on the Higgs boson properties and the inflationary parameters: BBN and CMB constraints}

For a robust interpretation of upcoming high precision temperature and polarization $\mathrm{CMB}$ anisotropies (Mandolesi et al., 2010), it is imperative to understand how the inflationary dynamics of a non-minimally coupled Higgs scalar field ( $\xi$-inflation) may affect the degeneracy of the inflationary observables, the determination of the Higgs boson mass and of the vacuum expectation value. 

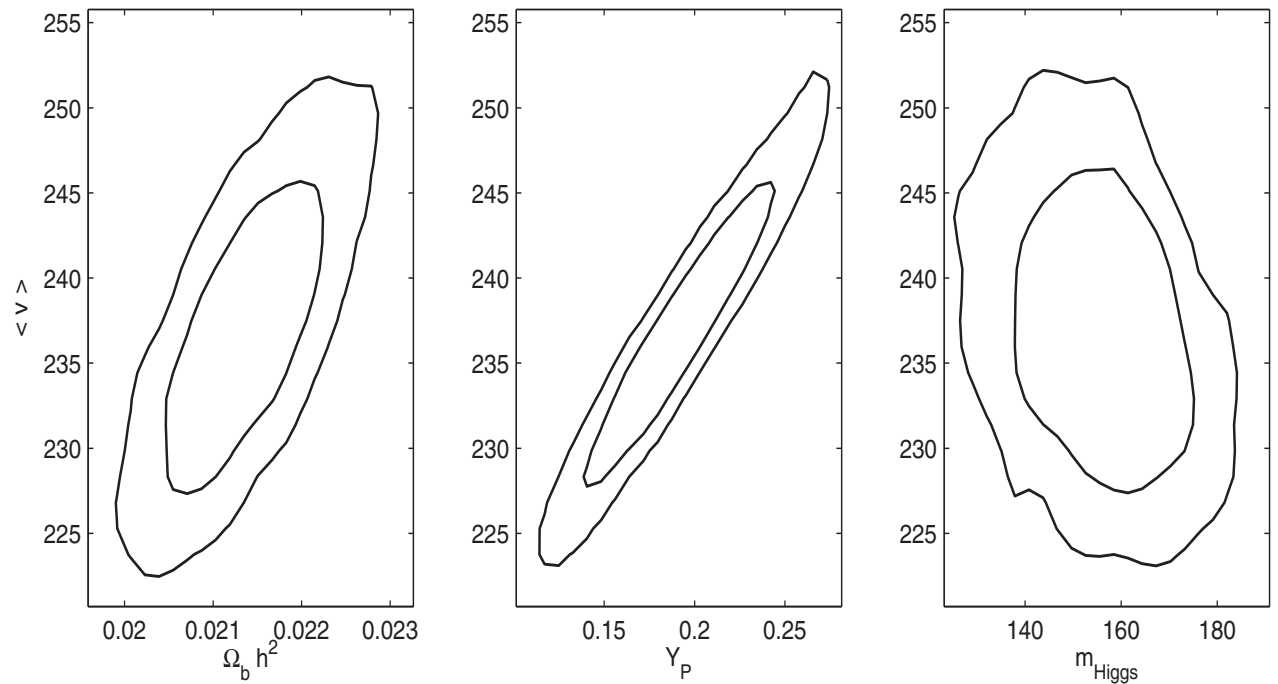

Fig. 4. Two dimensional joint probability distributions (68\% and 95\% CL) showing the degeneracy between different cosmological parameters as obtained from the fit of Higgs inflation model to the WMAP7+BBN+All data set.

\begin{tabular}{lcc}
\hline \hline $\begin{array}{l}\text { Model } \\
\text { Parameter }\end{array}$ & Standard Inflation Higgs Inflation \\
\hline $100 \Omega_{b} h^{2}$ & $2.259 \pm 0.054$ & $2.257 \pm 0.051$ \\
$\Omega_{c} h^{2}$ & $0.113 \pm 0.003$ & $0.114 \pm 0.003$ \\
$\tau$ & $0.088 \pm 0.015$ & $0.086 \pm 0.013$ \\
$\theta_{S}$ & $1.038 \pm 0.002$ & $1.037 \pm 0.002$ \\
$Y_{P}$ & $0.245 \pm 0.014$ & $0.247 \pm 0.091$ \\
$m_{H i g g s}(\mathrm{GeV})$ & - & $155.372 \pm 3.851$ \\
$\leq v>(\mathrm{GeV})$ & - & $243.131 \pm 5.912$ \\
\hline \hline
\end{tabular}

Table 2. The mean values from the posterior distributions of the parameters obtained from the fit of the standard inflation model and Higgs inflation model with $m_{T o p}=171.3 \mathrm{GeV}$ to the WMAP7+BBN+All dataset. The errors are quoted at $68 \% \mathrm{CL}$. All parameters are computed at the Hubble radius crossing $k_{*}=0.002 \mathrm{Mpc}^{-1}$.

We place bounds on these parameters by using WMAP7+BBN+All data set, in the context of the non-minimally coupled Higgs inflaton field with gravity.

We obtain the $\mathrm{CMB}$ temperature anisotropy and polarization power spectra by integrating the coupled Friedmann equation and equation of motion of the Higgs scalar field with respect to the conformal time (Popa \& Caramete, 2010; Popa, 2011).

The quantum corrections due to the interaction effects of the SM particles with Higgs boson through quantum loops modify Higgs potential from its classical expression. We consider the 
quantum corrections to the Higgs potential by including in the computation the running of the different coupling constants: the $\mathrm{SU}(2) \times \mathrm{SU}(1)$ gauge couplings $\left\{g^{\prime}, g\right\}$, the $S U(3)$ strong coupling $g_{s}$, the top Yukawa coupling $y_{t}$, and the Higgs quadratic coupling $\lambda$ (Espinosa et al., 2008).

At the top quark mass scale $m_{T o p}$, the Higgs quadratic coupling $\lambda(0)$ and the top Yukawa coupling $y_{t}(0)$ are determined by the corresponding Higgs boson and top quark pole masses and the vacuum expectation value $\langle v\rangle$ :

$$
\lambda(0)=\frac{m_{\text {Higgs }}^{2}}{2<v\rangle^{2}}\left[1+2 \Delta_{H}\left(m_{\text {Higgs }}\right)\right], \quad y_{t}(0)=\frac{\sqrt{2} m_{\text {Top }}}{\langle v\rangle}\left[1+\Delta_{T}\left(m_{\text {Top }}\right)\right],
$$

where $\Delta_{H}\left(m_{\text {Higgs }}\right)$ and $\Delta_{T}\left(m_{\text {Top }}\right)$ are the corrections to Higgs and top quark mass respectively. The likelihood probabilities are evaluated by using the public packages COSMOMC and CAMB (Lewis et al., 2000; Lewis \& S. Briddle, 2002) modified to include the formalisms for the SM Higgs driven $\xi$-inflation.

Our fiducial model is the $\Lambda \mathrm{CDM}$ cosmological model described by the following set of parameters receiving uniform priors:

$$
\Theta=\left\{\Omega_{b} h^{2}, \Omega_{c} h^{2}, \theta_{s}, \tau, m_{\text {Higgs }},<v>\right\}
$$

where: $\Omega_{b} h^{2}$ is the physical baryon density, $\Omega_{c} h^{2}$ is the physical dark matter density, $\theta_{s}$ is the ratio of the sound horizon distance to the angular diameter distance, $\tau$ is the reionization optical depth, $m_{\text {Higgs }}$ is the Higgs boson pole mass, $\langle v\rangle$ is the Higgs vacuum expectation value. As in the previous case, we use the PArthENoPE code (Pisanti et al., 2008) to obtain the ${ }^{4} \mathrm{He}$ mass fraction as function of $\Omega_{b} h^{2}$ and $\langle v\rangle$.

Figure 3 presents the marginalized posterior likelihood probability distributions of the main cosmological parameters and Figure 4 presents the two-dimensional joint probability distributions showing the degeneracy in the planes $\langle v\rangle-\Omega_{b} h^{2},\langle v\rangle-Y_{P}$ and $\langle v\rangle-$ $m_{\text {Higgs }}$, as obtained from the fit of the Higgs $\xi$-inflation model to the WMAP7+BBN+All data set.

Table 2 presents the mean values from the posterior distributions of the parameters obtained from the fit of the standard inflation model and Higgs inflation model with $m_{T o p}=171.3 \mathrm{GeV}$ to the WMAP7+BBN+All dataset. The errors are quoted at $68 \% \mathrm{CL}$.

From this analysis we obtain the vacuum expectation value and the Higgs boson mass in the limits expected from the collider experiments, while the cosmological parameters are compatible to the standard inflation scenario (Popa \& Caramete, 2009).

\section{Acknowledgments}

This work was partially supported by CNCSIS Contract 539/2009 and by ESA/PECS Contract C98051.

\section{References}

Abazajian, K. N.; Beacom, J. F.; Bell, N. F. (2002). Stringent Constraints on Cosmological Neutrino-Antineutrino Asymmetries from Synchronized Flavor Transformation. Phys. Rev. D 66, 013008 [astro-ph/0203442] 
Barvinsky, A. O.; Kamenshchik, A. Yu.; Starobinsky A. A. (2008). Inflation scenario via the Standard Model Higgs boson and LHC. JCAP 11, 021 [arXiv:0809.2104]

Bergstrom, L.; Iguri, S.; Rubinstein, H. (1999). Constraints on the variation of the fine structure constant from big bang nucleosynthesis. Phys. Review D 60, 045005 [arXiv:astro-ph/9902157]

Bezrukov, F. \& Shaposhnikov, M. (2008). The Standard Model Higgs boson as the inflaton. Phys. Lett. B 659, 703 [arXiv:0710.3755]

Bezrukov, F. L.; Magnin, A.; Shaposhnikov, M. (2009). Standard Model Higgs boson mass from inflation. Phys. Lett. B 675, 88 [arXiv:0812.4950]

Bezrukov, F.; Gorbunov, D.; Shaposhnikov, M. (2009). On initial conditions for the Hot Big Bang. JCAP 06, 029 [arXiv:0812.3622]

Boesgaard, A. M.; Steigman, G. (1985). Big bang nucleosynthesis - Theories and observations. Ann. Rev. Astron. Astrophys., 23, 319

Buchmuller, W.; Di Bari, P.; Plumacher, M. (2004). Some Aspects of Thermal Leptogenesis. New Jour. Phys. 6, 105 [hep-ph/0406014]

Burles, S.; Nollett, K. M.; Turner, M. S. (2001). Big-Bang Nucleosynthesis Predictions for Precision Cosmology. Astrophys. J. 552, L1 [astro-ph/0010171]

Chu, Y. Z. \& Cirelli, M. (2006). Sterile neutrinos, lepton asymmetries, primordial elements: How much of each? Phys. Rev. D 74, 085015

de Simone, A.; Hertzberg, M. P.; Wilczek, F. (2009). Running Inflation in the Standard Model. Phys. Lett. B 678, 1 [arXiv:0812.4946]

Dunkley, J. et al. (2009). Five-Year Wilkinson Microwave Anisotropy Probe (WMAP) Observations: Likelihoods and Parameters from the WMAP data. Astrophys. J. Supp. 180, 306 [arXiv:0803.0586]

Dolgov, A. D.; Hansen, S. H.; Pastor, S.; Petcov, S. T.; Raffelt, G.; Semikoz, D. V. (2002). Cosmological bounds on neutrino degeneracy improved by flavor oscillations. Nucl.Phys. B 632, 363 [hep-ph/0201287]

Eidelman S. et al. (2004). Review of particle physics. Phys. Lett. B 592, 1

Epstein, R.; Lattimer, J. \& Schramm, D. N. (1976). The origin of deuterium. Nature, 263, 198

Espinosa, J. R.; Giudice, G. F.; Riotto, A. (2008). Cosmological implications of the Higgs mass measurement. JCAP 05, 002 [arXiv:0710.2484]

Falcone, D. \& Tramontano, F. (2001). Neutrino oscillations and neutrinoless double beta decay. Phys. Rev. D 64, 077302 [hep-ph/0102136]

Freese, K.; Kolb, E. W.; Turner, M. S. (1983). Massive, degenerate neutrinos and cosmology. Phys. Rev. D27, 1689

Hinshaw et al. (2007). Three-Year Wilkinson Microwave Anisotropy Probe (WMAP) Observations: Temperature Analysis. Astrophys. J. Suppl. 170, 288 [astro-ph/0603451]

Hu, W.; Scott, D.; Sugiyama, N.; White, M. (1995). The Effect of Physical Assumptions on the Calculation of Microwave Background Anisotropies. Phys. Rev. D 52, 5498 [astro-ph/9505043]

Ichikawa, K.; Kawasaki, M.; Nakayama, K.; Senami, M., Takahashi, F. (2007). Increasing the effective number of neutrinos with decaying particles. JCAP 05, 008 [hep-ph/0703034]

Ichiki, K.; Yamaguchi, M.; Yokoyama, J. (2007). Lepton asymmetry in the primordial gravitational wave spectrum. Phys.Rev D 75, 084017 [hep-ph/0611121]

Izotov, Y. I. \& Thuan, T. X. (2004) Systematic effects and a new determination of the primordial abundance of $4 \mathrm{He}$ and $\mathrm{dY} / \mathrm{dZ}$ from observations of blue compact galaxies. Astrophys. J. 602, 200 [astro-ph/0310421] 
Izotov, Y. I.; Thuan, T. X.; Stasinska, G. (2007). The primordial abundance of 4He: a self-consistent empirical analysis of systematic effects in a large sample of low-metallicity HII regions. Astrophys. J. 662, 15 [astro-ph/0702072]

Komatsu E. et al. (2009). Five-Year Wilkinson Microwave Anisotropy Probe (WMAP) Observations: Cosmological Interpretation. Astrophys. J. Suppl. 180, 330 [arXiv:0803.0547 [astro-ph]].

Komatsu, E. et al. (2011). Seven-Year Wilkinson Microwave Anisotropy Probe (WMAP) Observations: Cosmological Interpretation. Astrophys. J. Suppl. 192, 18 [arXiv:1001.4538 [astro-ph.CO]]

Kowalski, M. et al. (2008). Improved Cosmological Constraints from New, Old and Combined Supernova Datasets. ApJ 686, 749 [arXiv:0804.4142 [astro-ph]]

Kuzmin, V.; Rubakov, V.; Shaposhnikov, M. (1985). On anomalous electroweak baryon-number non-conservation in the early universe. Phys. Lett. B 155, 36

Landau, S. J.; Mosquera, M. E.; Scoccola, C. G.; Vucetich, H. (2008). Early universe constraints on time variation of fundamental constants. Phys. rev. D 78, 083527 [arXiv:0809.2033]

Larson, D. et al. (2011). Seven-Year Wilkinson Microwave Anisotropy Probe (WMAP) Observations: Power Spectra and WMAP-Derived Parameters. Astrophys. J. Suppl. 192, 16 [arXiv:1001.4635 [astro-ph.CO]]

Lattanzi, M.; Ruffini, R.; Vereshchagin, G. V. (2005) Joint constraints on the lepton asymmetry of the Universe and neutrino mass from the Wilkinson Microwave Anisotropy Probe. Phys. Rev. D 72, 063003 [astro-ph/0509079]

Lewis, A.; Challinor, A.; Lasenby, A. (2000). Efficient Computation of Cosmic Microwave Background Anisotropies in Closed Friedmann-Robertson-Walker Models. ApJ 538, $473^{1}$ [arXiv:astro-ph/9911177]

Lewis, A. \& Briddle S. (2002). Cosmological parameters from CMB and other data: a Monte-Carlo approach. Phys. Rev. D 66, $103511^{2}$ [arXiv:astro-ph/0205436]

Mandolesi, N. et al. (Planck Collaboration) (2010). Planck pre-launch status: The Planck-LFI programme. Astron. E Astrophys. 520, A3 [arXiv:1001.2657 [astro-ph.CO]]

Mangano, G.; Miele, G.; Pastor, S.; Peloso, M. (2002). A precision calculation of the effective number of cosmological neutrinos. Phys. Lett. B 534, 8 [astro-ph/0111408]

Mangano, G.; Melchiorri, A.; Mena, O.; Miele, G.; Slosar, A. (2007). Present bounds on the relativistic energy density in the Universe from cosmological observables. JCAP 03, 006 [astro-ph/0612150]

Olive, K. A.; Steigman, G.; Walker, T. P. (2000). Primordial Nucleosynthesis: Theory and Observations. Phys. Rep. 333, 389 [astro-ph/9905320]

Olive, K. A. \& Skillman, E. D. (2004). A Realistic Determination of the Error on the Primordial Helium Abundance: Steps Toward Non-Parametric Nebular Helium Abundances. Astrophys. J. 617, 29 [astro-ph/0405588]

Page, L. et al. (2007) Three Year Wilkinson Microwave Anisotropy Probe (WMAP) Observations: Polarization Analysis. Astrophys. J.Suppl. 170, 335 [astro-ph/0603450]

W. J. Percival, et al. (2010). Baryon Acoustic Oscillations in the Sloan Digital Sky Survey Data Release 7 Galaxy Sample. MNRAS 401, 2148 [arXiv:0907.1660 [astro-ph]]

Pettini, M.; Zych, B. J.; Murphy, M. T.; Lewis, A.; Steidel, C. C. (2008). Deuterium abundance in the most metal-poor damped Lyman alpha system: converging on $O_{b, 0} h^{2}$. MNRAS 391, 1499 [arXiv:0805.0594 [astro-ph.CO]]

\footnotetext{
$\overline{{ }^{1} \text { http://camb.info }}$

${ }^{2}$ http:// cosmologist.info/cosmomc/
} 
Pisanti, O.; Cirillo, A.; Esposito, S.; Iocco, F.; Mangano, G.; Miele, G.; Serpico, P. D. (2008). PArthENoPE: Public algorithm evaluating the nucleosynthesis of primordial elements. Computer Physics Communications 178, 956 [arXiv:0705.0290 [astro-ph.CO]]

Popa, L. A. \& Vasile, A. (2008). WMAP 5-year constraints on lepton asymmetry and radiation energy density: Implications for Planck. JCAP 06, 028 [arXiv:0804.2971 [astro-ph.CO]]

Popa, L.A. \& Caramete, A. (2009). From WMAP to Planck: Exact Reconstruction of Fourand Five-dimensional Inflationary Potential from High-precision Cosmic Microwave Background Measurements. ApJ 706,1008 [arXiv:0907.5558 [astro-ph.CO]]

Popa, L.A. \& Caramete, A. (2010). Cosmological Constraints on the Higgs Boson Mass. ApJ 723,803 [arXiv:1009.1293 [astro-ph.CO]]

Popa, L.A. (2011). Observational consequences of the Standard Model Higgs inflation variants. JCAP 10, 025 [arXiv:1107.3436 [astro-ph.CO]]

Riess et al. (2009). A Redetermination of the Hubble Constant with the Hubble Space Telescope from a Differential Distance Ladder. ApJ 699, 539 (2009) [arXiv:0905.0695 [astro-ph.CO]]

Ruffini, R.; Song, D. J.; Stella, L. (1983). On the statistical distribution off massive fermions and bosons in a Friedmann universe. Astron. Astrophys. 125, 265

Ruffini, R.; Song, D. J.; Taraglio, S. (1988). The 'ino' mass and the cellular large-scale structure of the universe. Astron. Astrophys. 190, 1

Sarkar, S. (1996). Big bang nucleosynthesis and physics beyond the standard model. Rep. Prog. Phys. 59, 1493 [arXiv:hep-ph/9602260]

Serpico, P. D. \& Raffelt, G. G. (2005) Lepton asymmetry and primordial nucleosynthesis in the era of precision cosmology. Phys.Rev. D 71, 127301 [astro-ph/0506162]

Smith, C. J.; Fuller, G. M.; Kishimoto, C. T.; Abazajian, K. N. (2006). Light Element Signatures of Sterile Neutrinos and Cosmological Lepton Numbers. Phys. Rev. D 74, 085008 [astro-ph/0608377]

Spergel, D. N. et al. (2007). Wilkinson Microwave Anisotropy Probe (WMAP) Three Year Results: Implications for Cosmology. Astrophys. J. Suppl. 170, 377 [astro-ph/0603449]

Steigman, G.; Schramm, D. N.; Gunn, J. E. (1977). Cosmological limits to the number of massive leptons. Phys. Lett. B 66, 202

Steigman, G. (2007). Primordial Nucleosynthesis in the Precision Cosmology Era. Ann. Rev. Nucl. Part. Sci. 57, 463

Trotta, R. \& Melchiorri, A. (2005). Indication for Primordial Anisotropies in the Neutrino Background from WMAP and SDSS. Phys. Rev Lett. 95, 011305 [astro-ph/0412066]

Yoo, J. J. \& Scherrer, R. J. (2003). Big bang nucleosynthesis and cosmic microwave background constraints on the time variation of the Higgs vacuum expectation value. Phys. Rev. D 67, 043517 [arXiv:astro-ph/0211545]

Wagoner R. V.; Fowler W. A.; Hoyle F. (1967). On the Synthesis of Elements at Very High Temperatures. Astrophys. J. 148, 3

Wong, Y. Y. Y. (2002). Analytical treatment of neutrino asymmetry equilibration from flavour oscillations in the early universe. Phys. Rev. D 66, 025015 [hep-ph/0203180] 


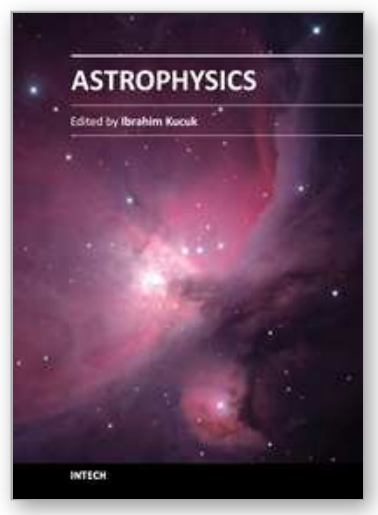

\author{
Astrophysics \\ Edited by Prof. Ibrahim Kucuk
}

ISBN 978-953-51-0473-5

Hard cover, 398 pages

Publisher InTech

Published online 30, March, 2012

Published in print edition March, 2012

This book provides readers with a clear progress to theoretical and observational astrophysics. It is not surprising that astrophysics is continually growing because very sophisticated telescopes are being developed and they bring the universe closer and make it accessible. Astrophysics Book presents a unique opportunity for readers to demonstrate processes do occur in Nature. The unique feature of this book is to cover different aspects in astrophysics covering the topics: • Astronomy • Theoretical Astrophysics • Observational Astrophysics • Cosmology • The Solar System • Stars • Planets • Galaxies • Observation • Spectroscopy • Dark Matter • Neutron Stars • High Energy Astrophysics

\title{
How to reference
}

In order to correctly reference this scholarly work, feel free to copy and paste the following:

L. A. Popa and A. Caramete (2012). BBN as Probe of Fundamental Physics, Astrophysics, Prof. Ibrahim Kucuk (Ed.), ISBN: 978-953-51-0473-5, InTech, Available from: http://www.intechopen.com/books/astrophysics/bbnas-probe-of-fundamental-physics

\section{INTECH}

open science | open minds

\section{InTech Europe}

University Campus STeP Ri

Slavka Krautzeka 83/A

51000 Rijeka, Croatia

Phone: +385 (51) 770447

Fax: +385 (51) 686166

www.intechopen.com

\section{InTech China}

Unit 405, Office Block, Hotel Equatorial Shanghai

No.65, Yan An Road (West), Shanghai, 200040, China

中国上海市延安西路65号上海国际贵都大饭店办公楼405单元

Phone: +86-21-62489820

Fax: +86-21-62489821 
(C) 2012 The Author(s). Licensee IntechOpen. This is an open access article distributed under the terms of the Creative Commons Attribution 3.0 License, which permits unrestricted use, distribution, and reproduction in any medium, provided the original work is properly cited. 